\title{
Resistance of $\beta$-lactamase-producing Klebsiella pneumoniae to Imipenem with Ompk36 loss
}

\author{
M. J. Jiang, Sh. P. Zhao* and J. M. Li \\ Tai An City Central Hospital, Shan Dong Province, China. \\ Accepted 26 January, 2012
}

\begin{abstract}
The present study investigated the mechanisms involved in decreased imipenem susceptibility in Klebsiella pneumoniae. Pulsed field gel electrophoresis was used for homology analysis, and a modified Hodge test was used to detect carbapenemase expression. An ethylenediaminetetraacetic acid (EDTA) synergy test was used to screen for metallo- $\beta$-lactamase. An E-test and a conjugation experiment were used to detect drug susceptibility, whereas specific polymerase chain reaction (PCR) amplification and sequencing, sodium dodecyl sulfate polyacrylamide gel electrophoresis (SDS-PAGE) and carbonyl cyanide-m-chlorophenylhydrazone (CCCP) inhibition tests were used to analyze mechanisms underlying the decrease in imipenem susceptibility in $K$. pneumoniae. $\mathrm{Kp} 1, \mathrm{Kp2}$, and $\mathrm{Kp3}$ did not belong to the same clone strain and their minimum inhibitory concentrations for imipenem and meropenem were 32, 4, and $24 \mu \mathrm{g} / \mathrm{ml}$ and 4,8 , and $4 \mu \mathrm{g} / \mathrm{ml}$, respectively. $\mathrm{Kp} 1$ and $\mathrm{Kp} 3$ were resistant to piperacillin/tazobactam, whereas $\mathrm{Kp2}$ was not. $\mathrm{Kp} 1, \mathrm{Kp} 2$, and $\mathrm{Kp} 3$ were highly resistant to cephalosporins and aztreonam. Kp2 and Kp3 were resistant to gentamicin. All produced $\beta$-lactamase DHA-1, CTX-M-14, SHV-12, and KPC-2. Kp3 produced CMY-2 and cephalosporinase. Gene sequencing analysis and outer membrane porin SDS-PAGE showed that the Ompk36 gene of Kp1 and Kp3 was inserted by IS5 and IS903, which resulted in the loss of the outer membrane porin Ompk36. CCCP did not increase the sensitivity of $\mathrm{Kp} 1, \mathrm{Kp} 2$, and $\mathrm{Kp} 3$ to imipenem. Therefore, the loss of the outer membrane porin Ompk36 in $\beta$-lactamase KPC-2, DHA-1, CTX-M-14 and SHV-12 producing $K$. pneumoniae induces the development of high-level imipenem resistance.
\end{abstract}

Key words: Klebsiella pneumoniae, $\beta$-lactamase, imipenem, drug sensitivity, outer membrane porin.

\section{INTRODUCTION}

Klebsiella pneumoniae is one of the most common bacteria that cause nosocomial infections with multiple drug resistance because of its plasmid-mediated $\beta$ lactamase. Extended spectrum beta-lactamases (ESBLs) and $\mathrm{AmpC}$ occasionally coexist, which lead to drug resistance to many antibiotics (Moland et al., 2002). Carbapenem antibiotics are the drug of choice for treating severe infections caused by Enterobacteriaceae bacteria because of their high stability against ESBLs and AmpC, as well as strong bacterial activity (Ye et al., 2005). Thus, the use of carbapenem-resistant Enterobacteriaceae bacteria has attracted increasing attention. The first

${ }^{*}$ Corresponding author. E-mail: I.jm.2007@hotmail.com. Tel: +86-0538-2138372. Fax: +86-0538-2138372. carbapenemase-producing $K$. pneumoniae KPC-1 was reported in 2001 (Yigit et al., 2001), K.pneumoniae carbapenemase (KPC) was successively developed in USA, South America, and China (Villegas et al., 2006; Wei and Yu, 2007; Navon-Venezia et al., 2006; Zhang et al., 2006). KPC is a Class A carbapenemase whose resistance genes spread between different bacteria with epidemic risks (Woodford et al., 2004; Yigit et al., 2003; Zhang et al., 2008). K. pneumoniae that produce AmpC combined with loss of outer membrane porins which lead to resistance or mediates resistance to carbapenems (Bradford et al., 1997; Zhang et al., 2008). K. pneumoniae that produces AmpC, CMY-4 (Cao et al., 2000 ) or SHV-2 (Crowley et al., 2002) combined with loss of outer membrane porins lead to resistance or mediate resistance to carbapenems, which has also been reported in NDM-1-producing $K$. pneumoniae. NDM-1- 
producing bacteria are resistant to many antibiotics, which makes their clinical treatment difficult. We analyzed the strains $\mathrm{Kp} 1, \mathrm{Kp} 2$, and $\mathrm{Kp} 3$, which have different homologies but produce the same $\beta$-lactamase, to understand the resistance mechanisms of $K$. pneumoniae to imipenem.

\section{MATERIALS AND METHODS}

\section{Bacterial isolates}

Kp1 was highly resistant to imipenem isolated from the sputum of an incubated child in the pediatric ward in June 2006. The patient was a neonate diagnosed with pneumonia, wet lung, and respiratory failure. After empiric treatment with ceftriaxone for $4 \mathrm{~d}$, imipenem was administered for $5 \mathrm{~d}$, and $\mathrm{Kp} 1$ was isolated from the sputum during incubation. Kp2, which has low resistant to imipenem, was isolated from the sputum of a neonatal patient diagnosed with pneumonia after an 8 day treatment with panipenem. $\mathrm{Kp} 3$ is a highly imipenem-resistant isolate from the sputum of a 79-year-old man clinically diagnosed with acute myocardial infarction and pneumonia. Kp3 was isolated after treatment with ceftriaxone and levofloxacin for 3 days and then with panipenem for 15 days. The negative control strain was Escherichia coli ATCC25922. The control strain of ATCC13883 was used for outer membrane porin analysis. The KPC-positive standard strain was provided by Dr. Shen Jilu Shen et al. (2008).

\section{Pulsed field gel electrophoresis (PFGE)}

PFGE was designed according to standard methods done by the CDC (Pulse Net USA, 2002). A single bacterial colony was inoculated into the lysogeny broth (LB) medium and stored overnight under vigorous agitation. Bacilli mixed with low melting point plastic were added into mold and incubated with proteinase $\mathrm{K}$ for $48 \mathrm{~h}$. The mixture was washed with TE buffer and incubated overnight with XBA I endonulease. Then, 1\% agarose gel was prepared, and PFGE was performed using CHEF-DR III (Bio-Rad, USA). The electrophoresis parameters were as follows: $6 \mathrm{~V} / \mathrm{cm}$, pulse interval time of $3 \mathrm{~s}$ to $40 \mathrm{~s}, 0.5 \times$ tris-borate-EDTA (TBE) electrophoresis buffer, electric field angle of $120^{\circ} \mathrm{C}$, and electrophoresis time of $24 \mathrm{~h}$.

\section{Modified hodge test}

Around $0.5 \mathrm{Mx}$ of $E$. coli ATCC25922 suspension was coated onto the $\mathrm{MH}$ plate. A slip of ertapenem was placed in the middle of the $\mathrm{MH}$ plate. Around 3 to 5 colonies were inoculated as a straight line from the slip edge to the flat edge, and incubated for 16 to $20 \mathrm{~h}$ at $35^{\circ} \mathrm{C}$.

\section{EDTA synergy test}

Around 0.5 Mx of E. coli ATCC25922 suspension was coated onto the $\mathrm{MH}$ plate. A slip of imipenem was coated $1.0 \mathrm{~cm}$ away from the blank slip coating. Around $4 \mu \mathrm{l}$ of $0.5 \mathrm{~mol} / \mathrm{L}$ EDTA was placed above the blank slip, and incubated for 16 to $20 \mathrm{~h}$ at $35^{\circ} \mathrm{C}$.

\section{Antibiotic susceptibility testing}

E-test was used to determine drug resistance of strains to imipenem, meropenem, ertapenem, piperacillin/tazobactam, aztreonam, cefotaxime, ceftazidime, cefepime, cefoxitin, levofloxacin, amikacin and gentamicin. Antimicrobial susceptibility was determined using CLSI, 2011(Clinical and Laboratory Standards Institute, 2011).

\section{Plasmid conjugation test}

Plasmid conjugation experiment was done as previously described (Zhang et al., 2008). E. coli J53 was the recipient strain, and Kp1, $\mathrm{Kp} 2$, and Kp3 were the donor strains. Conjugons were identified using the ATB Bacteria Identification System.

PCR amplification and electrophoresis of KPC, ESBLs, AmpC, and NDM-1 genes

The primers were designed as previously described (Yong et al., 2009; Kumarasamy et al., 2010).

The PCR primers for KPC, ESBLs, AmpC, and NDM-1 are shown in Table 1. PCR was carried out as previously described (NavonVenezia et al., 2006). The PCR products were electrophoresed in agarose gel containing $2 \%$ ethidium bromide, and a gel imaging system was used to observe the result.

\section{SDS-PAGE analysis}

The outer membrane porins of $\mathrm{Kp} 1, \mathrm{Kp} 2, \mathrm{Kp} 3$, and the standard strain ATCC13883 were extracted as previously described (Zhang et al., 2008). Band differences between the standard and clinical $K$. pneumoniae strains were compared.

PCR amplification and DNA sequencing of the Ompk35 and Ompk36 genes

The PCR primers of Ompk35 and Ompk36 were designed as previously described (Zhang et al., 2008). The OmpK35 and OmpK36 genes of $\mathrm{Kp} 1, \mathrm{Kp} 2$, and $\mathrm{Kp} 3$ were detected using PCR. The PCR amplification products were compared with the standard sequence (GenBank accession number MH000060) after gene sequencing. The primer sequences are listed in Table 2.

\section{Efflux pump mechanism analysis}

A CCCP inhibition test was used to analyze whether imipenem caused an active efflux by $\mathrm{Kp} 1, \mathrm{Kp} 2$, and Kp3. The $\mathrm{MH}$ agar plates of imipenem concentration gradients (dilution from 32 to 0.125 $\mu \mathrm{g} / \mathrm{ml})$ with or without $50 \mu \mathrm{mol} / \mathrm{L} \mathrm{CCCP}^{12}$ were prepared to determine the minimum inhibitory concentrations (MIC) of the strains.

\section{RESULTS}

Drug susceptibility test showed that the MIC of Kp1, Kp2, and $\mathrm{Kp} 3$ to imipenem and meropenem were 32,4 , and $24 \mu \mathrm{g} / \mathrm{ml}$ and 4,8 , and $4 \mu \mathrm{g} / \mathrm{ml}$, respectively. The strains were highly resistant to ertapenem, ceftazidime, cefotaxime, aztreonam, amoxicillin/clavulanic acid, and cefoxitin, and have different degrees of resistance to cefepime. Kp2 was sensitive to piperacillin/tazobactam, whereas $\mathrm{Kp} 1$ and $\mathrm{Kp} 3$ were not. $\mathrm{Kp} 2$ and $\mathrm{Kp} 3$ were 
Table 1. PCR primers of gene KPC, ESBLs, AmpC and NDM-1.

\begin{tabular}{|c|c|c|c|}
\hline Primers & Sequence $\left(5^{\prime} \rightarrow 3^{\prime}\right)$ & Length of the products (bp) & Annealing temperature $\left({ }^{\circ} \mathrm{C}\right)$ \\
\hline SHV-F & GGTTATGCGTTATATTCGCC & $867^{\mathrm{bp}}$ & 55 \\
\hline SHV-R & TTAGCGTTGCCAGTGCTC & & \\
\hline CTX-M1-F & AGTGCAAACGGATGATGT & $792^{\text {bp }}$ & 55 \\
\hline CTX-M1-R & GGCTGGGTAAAAATAGGTC & & \\
\hline CTX-M2-F & ACGCTACCCСTGCTATT & $830^{\mathrm{bp}}$ & 55 \\
\hline CTX-M2-R & CAGAAACCGTGGGTTACGA & & \\
\hline CTX-M3-F & ACGCTGTTGTTAGGAAGTG & $759^{\mathrm{bp}}$ & 55 \\
\hline CTX-M3-R & TTGAGGCTGGGTGAAGT & & \\
\hline DHA-MF & AACTTTCACAGGTGTGCTGGGT & $405^{\mathrm{bp}}$ & 55 \\
\hline DHA-MR & CCGTACGCATACTGGCTTTGC & & \\
\hline CTT-MF & TGGCCAGAACTGACAGGCAAA & $462^{\mathrm{bp}}$ & 55 \\
\hline CTT-MR & TTTCTCCTGAACGTGGCTGGC & & \\
\hline ACC-MF & AACAGCCTCAGCAGCCGGTTA & $346^{\mathrm{bp}}$ & 55 \\
\hline ACC- MR & TTCGCCGCAATCATCCCTAGC & & \\
\hline KPC-gp-F & GCGGAACCATTCGCTAAACTC & $340_{\mathrm{bp}}$ & 55 \\
\hline KPC-gp-R & CGCCCAACTCCTTCAGCAACA & & \\
\hline KPC-qc-F & ATGTCACTGTATCGCCGTCT & $882^{\mathrm{bp}}$ & 55 \\
\hline KPC-qc-R & TTACTGCCCGTTGACGC & & \\
\hline NDM-1-F & GGCGGAATGGCTCATCACGA & 286 & 60 \\
\hline NDM-1-R & CGCAACACAGCCTGACTTTC & & \\
\hline
\end{tabular}

Table 2. PCR primer sequence of ompK35 and ompK36.

\begin{tabular}{lllc}
\hline Gene & Primers & Sequence $\left(\mathbf{5}^{\prime} \rightarrow \mathbf{3}^{\prime}\right)$ & Length of the product (bp) \\
\hline ompK35 & ompK35-F & CAGACACCAAACTCTCATCAATGG & 1183 \\
ompK35-R & AGAATTGGTAAACGATACCCACG & 1115 \\
& $\begin{array}{l}\text { ompK36-F } \\
\text { ompK36-R }\end{array}$ & $\begin{array}{l}\text { CAGCACAATGAATATAGCCGAC } \\
\text { GCTGTTGTCGTCCAGCAGGTTG }\end{array}$ & \\
\hline
\end{tabular}

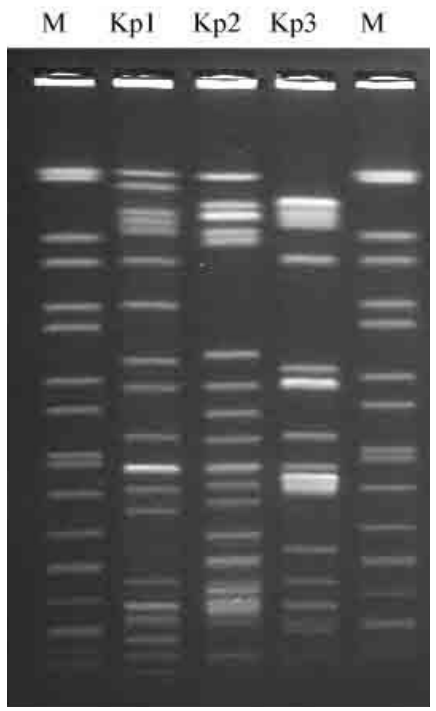

Figure 1. PFGE results of $\mathrm{Kp} 1$, $\mathrm{Kp} 2$, and Kp3 resistant to gentamicin, whereas $\mathrm{Kp} 1$ was not. $\mathrm{Kp} 1$ and Kp2 were sensitive to amikacin and levofloxacin, whereas Kp3 was not. The results of the PFGE Kp1, Kp2, and Kp3 are shown in Figure 1. The electrophoresis strips for Kp1, $\mathrm{Kp} 2$, and Kp3 had significant differences, which indicate that the three strains belong to different clones.

The Kp1 and Kp3 conjunction experiment was successful. The conjugons of Kp1 and Kp3 grew on the screen plates containing $100 \mu \mathrm{g} / \mathrm{ml}$ sodium azide and $8 \mu \mathrm{g} / \mathrm{ml}$ cefotaxime and in plates containing $100 \mu \mathrm{g} / \mathrm{ml}$ sodium azide and $0.5 \mu \mathrm{g} / \mathrm{ml}$ imipenem. The conjugon did not grow on either screen plate, which indicates that the conjugation experiment failed. The conjugons of $\mathrm{Kp} 1$ and Kp3 were sensitive to imipenem, meropenem, ertapenem, aztreonam, ceftazidime, cefotaxime, aztreonam, cefepime, piperacillin/tazobactam, levofloxacin, amikacin, and gentamicin while resistant to cefotaxime, amoxicillin/clavulanic acid, and cefoxitin. The drug susceptibility of ceftazidime was different in both $\mathrm{Kp} 1$ and $\mathrm{Kp} 3$. The MICs of $\mathrm{Kp} 1, \mathrm{Kp} 2$, and $\mathrm{Kp} 3$, as well as 
Table 3. MIC $(\mu \mathrm{g} / \mathrm{ml})$ of antibacterials and drug resistance gene of $\beta$-lactamase for donor bacterium, receptor bacterium, conjugon and passaged $\mathrm{Kp}$.

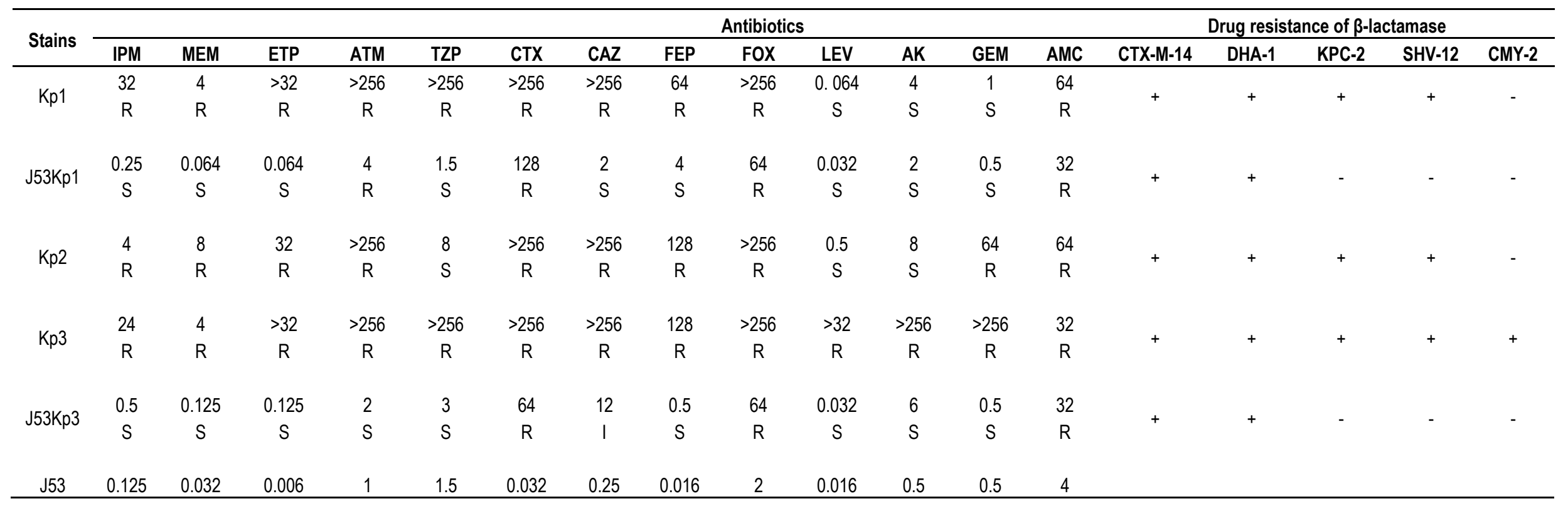

Note: R: resistance; I: Intermediary; S: Sensitive.

for J53Kp1 and J53Kp3, are shown in Table 3.The results of the modified Hodge test show that Kp1, $\mathrm{Kp} 2$, and Kp3 all produce carbapenemase.

The results from the EDTA synergy test show that $\mathrm{Kp} 1, \mathrm{Kp} 2$, and $\mathrm{Kp} 3$ did not produce metallo- $\beta$ lactamase.

The PCR amplification of drug resistance genes show that $\mathrm{Kp} 1, \mathrm{Kp} 2$, and $\mathrm{Kp} 3$ produced $\mathrm{DHA}-1$, CTX-M-14, SHV-12, and KPC-2. Kp3 also produced CMY-2. The conjugons of $\mathrm{Kp} 1$ and $\mathrm{Kp} 3$ contained DHA-1 and CTX-M-14. The conjugon of Kp1 could not produce SHV-12 and KPC 2, and the conjugon of $\mathrm{Kp} 3$ could not produce SHV-12, CMY-2, and KPC-2.

The SDS-PAGE results of $\mathrm{Kp} 1, \mathrm{Kp} 2, \mathrm{Kp} 3$, and the standard strain ATCC13883 outer membrane porins are shown in Figure 2. Kp1 and Kp3 lacked
Ompk36 compared with the standard strain ATCC13883. The target gene fragment of OmpK36 was detected in Kp2. OmpK36 gene fragments of Kp1 and Kp3 were 1200 bp larger than that of Kp2. IS5 and IS903 were inserted into $\mathrm{Kp} 1$ and Kp3, respectively, which led to abnormal gene expression. The target gene fragment of OmpK35 was detected in Kp1, Kp2, and Kp3.

The MICs of imipenem for Kp1, Kp2, and Kp3 were the same even with the addition of CCCP. Thus, Kp1, Kp2, and Kp3 had no active efflux mechanism for imipenem.

\section{DISCUSSION}

Since the first strain of ESBLs-producing $K$. pneumoniae was found in Germany in 1983, the development of multidrug-resistant $K$. pneumoniae has been a worldwide concern. Carbapenem antibiotics are the drug of choice for treating severe infections caused by Enterobacteriaceae bacteria because of their high stability to ESBLs and AmpC, as well as having a strong bacterial activity (Ye et al., 2007). The first imipenem-resistant $K$. pneumoniae (Kp-1) was isolated from the neonatal ward by our microbiology lab in June 2006. About 16 stains of imipenem-resistant $K$. pneumoniae have been isolated since 2009, which indicates that infections by imipenem-resistant $K$. pneumoniae have been increasing. Moreover, some clinical cases treated with carbapenem antibiotics have failed (such as patients infected by Kp3). To 


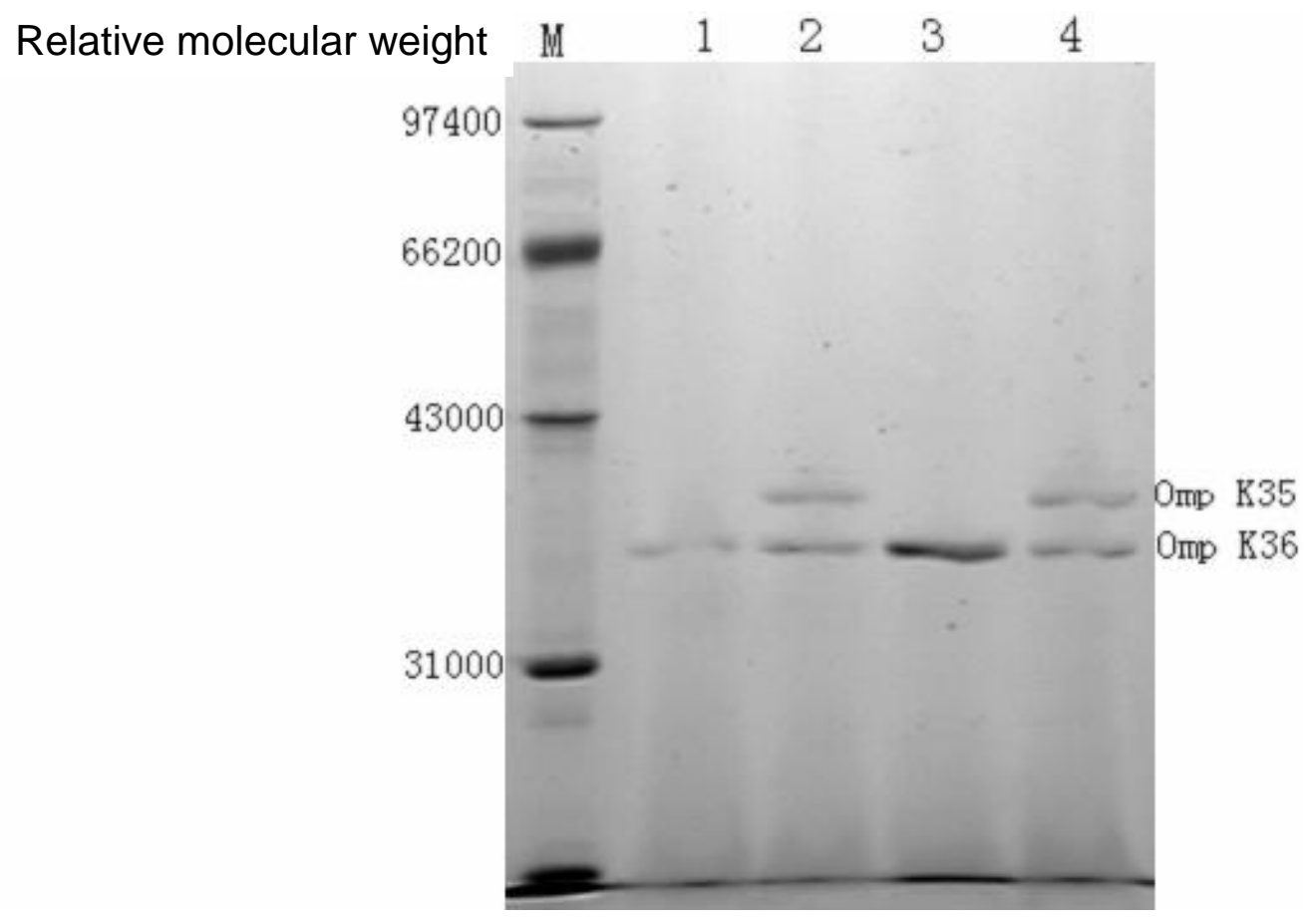

Figure 2. SDS-PAGE analysis of outer membrane porin $\mathrm{Kp} 1, \mathrm{Kp} 2$ and $\mathrm{Kp} 3 . \mathrm{M}$ stands for standard protein molecular weight; S stands for Kp ATCC13883.

investigate whether the decreased susceptibility of $K$. pneumoniae to imipenem prevents infections caused by multidrug-resistant strains and increase clinical efficacy. Cases of decreased susceptibility of $K$. pneumoniae to imipenem have been reported. Petrella et al. (2008) reported that the KPC-2 stain has low sensitivity to imipenem. The loss of an outer membrane porin combined with AmpC leads to resistance or mediation to carbapenem antibio-tics such as imipenem (DomenechSanchez et al., 1999; Lee et al., 2007). Enterobacteriaceae bacteria include $K$. pneumoniae, $E$. coli, and Enterobacter aerogenes, which may be resistant to imipenem because of the change in membrane permeability that results from the loss of outer membrane porins (Yigit et al., 2002; Stapleton et al., 1999). We conclude that Kp3 produces more CYM-2 AmpC than $\mathrm{Kp} 1$, but both increased the MIC of imipenem by the same degree. Thus, the main reason behind the increase in the MIC of imipenem is not due to CYM-2 AmpC. Crowley et al. (2002) reported the porin loss of Ompk35 and Ompk36 and that the ESBL SHV-2 results in a high resistance to meropenem (MIC: $16 \mu \mathrm{g} / \mathrm{ml}$ ). The mass production of AmpC (Bradford et al., 1997) and CMY-4 (Cao et al., 2000) or SHV-2 (Crowley et al., 2002), combined with the loss of outer membrane porins lead to resistance or intermediate resistance to carbapenem antibiotics.

We have isolated two $K$. pneumoniae strains containing CTX-M-14, KPC-2, DHA-1, IPM-4, and SHV-2. One the strains, which carry Ompk35 and Ompk36, only lead to intermediate resistance to imipenem (MIC: $1.5 \mu \mathrm{g} / \mathrm{ml}$ ). The other strain, which had no Ompk36, lead to low-level resistance to imipenem (MIC: $4 \mu \mathrm{g} / \mathrm{ml}$ ) and high-level resistance to meropenem (MIC: $16 \mu \mathrm{g} / \mathrm{ml}$ ).

The loss of Ompk36 and ESBL SHV-2 led to high resistance to imipenem (MIC: $32 \mu \mathrm{g} / \mathrm{ml}$ and $24 \mu \mathrm{g} / \mathrm{ml}$, respectively) and low resistance to meropenem (MIC: 4 $\mu \mathrm{g} / \mathrm{ml}$ ). The results in Table 3 show that $K$. pneumoniae that produces carbapanemase (KPC-2) and DHA-1 AmpC have strains that are both sensitive and resistant to piperacillin/tazobactam. The reason behind this behavior still needs further investigation.

We found that the conjugons J53Kp1 and J53Kp3, containing CTX-M-14 and DHA-1, have lowl resistant to cefoxitin (MIC: $64 \mu \mathrm{g} / \mathrm{ml}$ ), resistant to cefotaxime and amoxicillin/clavulanic acid, sensitive to cefotaxime, aztreonam, and piperacillin/tazobactam (Figure 1), which shows that the conjugons are sensitive to piperacillin/ tazobactam when DHA-1 expression is not high.

The results show that $K$. pneumoniae producing both ESBL SHV-12 and CTX-M-14, carbapenemase KPC-2, and AmpC DHA-1 without the loss of Ompk35 and Ompk36 induces low imipenem resistance. $K$. pneumoniae that produces SHV-12, CTX-M-14, carbapenemase KPC-2, and DHA-1 with the loss of AmpC and Ompk36 results in high imipenem resistance.

\section{REFERENCES}

Bradford PA, Urban C, Mariano N (1997). Imipenem resistance in 
Klebsiella pneumoniae is associated with the combination of ACT-1, a plasmid-mediated AmpC $\beta$-lactamase, and the loss of an outer membrane protein. Antimicrob. Agents Chemother., 41(3): 563-569.

Bradford PA, Urban C, Mariano N (1997). Imipenem resistance in Klebsiella pneumoniae is associated with the combination of ACT-1, a plasmid-mediated AmpC $\beta$-lactamase, and the loss of an outer membrane protein. Antimicrob. Agents Chemother., 41(3): 563-569.

Cao VT, Arlet G, Ericsson BM (2000). Emergence of imipenem resistance in Klebsiella pneumoniae owing to combination of plasmidmediated CMY-4 and permeability alteration. J. Antimicrob. Chemother., 46(6): 895-900.

Clinical and Laboratory Standards Institute (2011). M100-S21 Performance standards for antimicrobial susceptibility testing twentyfirst informational supplement [S]. 2011.

Crowley B, Benedi VJ, Domenech-Sanchez A (2002). Expression of SHV-2 $\beta$-lactamase and of reduced amounts of OmpK36 porin in Klebsiella pneumoniae results in incrased resistance to cephalosporins and carbapenems. Antimicrob. Agents Chemother., 46(11): 3679-3682.

Domenech-Sanchez A, Hernandez-Alles S, Martinez-Martinez L (1999). Identification and characterization of a new porin gene of Klebsiella pneumoniae: its role in $\beta$-lactam antibiotic resistance. J. Bacteriol., 181(9): 2726-2732.

Kumarasamy KK, Toleman MA, Walsh TR (2010). Emergence of a new antibiotic resistance mechanism in India, Pakistan and the UK: a molecular, biological and epidemiological study. Lancet Infect. Dis., 10:597-602

Lee K, Yong D, Choi YS (2007). Reduced imipenem susceptibility in Klebsiella pneumoniae clinical isolates with plasmid-mediated CMY-2 and DHA-1 $\beta$-lactamases co-mediated by porin loss. Int. J. Antimicrob. Agents, 29(2):201-206.

Moland ES, Black JA, Ourada J (2002). Thomson KS. Occurrence of newer beta-lactamases in Klebsiella pneumoniae isolates from $24 \mathrm{U}$. S. hospitals. Antimicrob. Agents Chemother., 46: 3837-3842.

Navon-Venezia S, Chmelnitsky I, Leavitt A (2006). Plasmid-mediated imipenem-hydrolyzing enzyme KPC-2 among muliple carbapenemresistant Escherichia coli clones in Israel. Antimicrob. Agents Chemother., 50(9): 3098-3101.

Petrella S, Ziental-Gelus N, Mayer C (2008). Genetic and structural insighis into the dissemination potential of the extremely broadspectrum class A beat-lactamase KPC-2 identified in an Escherichia coli strain and an Enterobacter cloacae strain isolated from the same patient in France. Antimicrob. Agents Chemother., 52(10): 3725-3736.

Shen JL, Zhu DM, Wu WH (2008). The relationship between acquired carbapenemases and resistance of Gram-negative bacill, Chin. J. Lab. Med., 31(4): 408-414.
Stapleton PD, Shannon KP, French GL (1999). Carbapanem resistance in Escherichia coli associated with plasmid-determined CMY-4 betalactamase production and loss of an outer membrane protein. Antimicrob. Agents Chemother., 43(5): 1206-1210.

Villegas MV, Lolans K, Correa A (2006). First detection of the plasmidmediated class A carbapenemase KPC-2 in clinical isolates of Klebsiella pneumoniae from South America. Antimicrob. Agents Chemother., 50(8): 2880-2882.

Wei ZQ, Yu YS (2007). Plasmid-mediated KPC-2 in a Klebsiella pneumoniae isolate from China. Antimicrob. Agents Chemother. 51(2): 763-765.

Woodford N, Tieno PM J, Young K (2004). Outbrek of Klebsiella pneumoniae producing a new carbappenem-hydrolyzing class $A \beta$ lactamase, KPC-3, in a New York Medical Center. Antimicrob. Agents Chemother., 48(12): 4793-4799.

Ye SJ, Yang Q, Yu YS (2007). CHINET 2005 surveillance of antimicrobial resistance in Escherichia coli and Klebsiella pneumoniae. Chin. J. Infect. Chemother., 7(4): 283-286.

Yigit H, Anderson GJ, Biddle JW (2002). Carbapenem resistance in a clinical isolate of Enterobacteraerogenesis associated with decrease dexpression of OmpF and OmpC porin analogs. Antimicrob. Agents Chemother., 46(12): 3817-3822.

Yigit H, Queenan AM, Anderson GJ (2001). Novel carbapenemhydrolyzing beta-lactamase, KPC-1, from a carbapenemresistant strain of Klebsiella pneumoniae. Antimicrob. Agents Chemother., 45(9): 1151-1161.

Yigit H, Queenan AM, Rasheed JK (2003). Carbapenem-resistant strain of Klebsiella oxytoca harboring carbapenem-hydrolyzing B-lactamase KPC-2. Antimicrob. Agents Chemother., 47(12): 3881-3889.

Yong D, Tolemon MA, Giske CG (2009). Characterization of a new metallo-beta-lactamase gene, bla(NDM-1), and a novel erythromycin esterase gene carried on a unique genetic structure in Klebsiella pneumoniae sequence type 14 from India. Antimicrob. Agents Chemother., 53: 5046-5054.

Zhang R, Cai JC, Zhou HW (2008). Identification of plasmid-mediated carbapenem-hydrolyzing $\beta$-lactamase KPC-2 in Enterobacteriaceae. Chin. J. Lab. Med., 31(10): 1134-1141.

Zhang R, Cai JC, Zhou HW (2008). Reduced carbapenem susceptibility in Klebsiella pneumoniae clinical isolates is due to combination of $\beta$ lactamases and the loss of OmpK36 porin, Chin. J. Microbiol. Immun., 28(1): 44-49.

Zhang XG, Du XX, Zhang RW (2006). Plasmid-mediated carbapenemase KPC-2 in a strain of Klebsieila pneumoniae. Chin. J. Lab. Med., 29: 824-826. 